PROCEEDINGS OF THE

AMERICAN MATHEMATICAL SOCIETY

Volume 137, Number 11, November 2009, Pages 3939-3942

S 0002-9939(09)09978-X

Article electronically published on July 13, 2009

\title{
A PARAMETRIZED FIXED POINT THEOREM
}

\author{
VESTA COUFAL \\ (Communicated by Brooke Shipley)
}

\begin{abstract}
We use bordism theory to extend Lefschetz-Nielsen theory to a family of manifolds and endomorphisms. In particular, we define an invariant, and prove a parametrized fixed point theorem and its converse.
\end{abstract}

\section{INTRODUCTION}

In Lefschetz-Nielsen fixed point theory, one considers an endomorphism $f: M \rightarrow$ $M$ of a compact manifold $M$. Briefly, one begins by choosing a base point $*$ and a base path $\tau$. A Lefschetz-Nielsen invariant, $L(f, *, \tau)$, can then be defined using the fundamental group and a trace construction [3]. Wecken proved the Hopf-Lefschetz theorem and its converse:

Theorem 1. When $M$ is a compact manifold of dimension greater than two, $L(f, *, \tau)=0$ if and only if $f$ is homotopic to a map with no fixed points.

Our goal is to extend Lefschetz-Nielsen theory to a family of manifolds and endomorphisms, i.e., a smooth fiber bundle $p: E \rightarrow B$ together with a map $f$ : $E \rightarrow E$ such that $p=p \circ f$. A simple extension of $L(f, *, \tau)$ is not possible because of the necessity of choosing a base point. In this paper, we avoid this problem and accomplish our goal by using bordism theory. See [5] and [1.

In Section 2 we give the definitions and results that will be needed in the construction of the invariant and in the proof of the parametrized fixed point theorem. In Section 3 we define the parametrized version of the Lefschetz-Nielsen invariant, and in Section 4 we state and prove the parametrized fixed point theorem.

\section{General Definitions And RESUlts}

In this section we give the definitions and results that we will need in our construction and proof. These are known but are included for the convenience of the reader.

Definition 2. The homotopy pullback of the diagram

$$
A \stackrel{g}{\longrightarrow} C \stackrel{h}{\longleftarrow} B
$$

of topological spaces and continuous maps is the space

$$
E(g, h)=\left\{(a, \theta, b) \in A \times C^{I} \times B \mid \theta \text { is a path in } C \text { from } g(a) \text { to } h(b)\right\} .
$$

Received by the editors March 18, 2009.

2000 Mathematics Subject Classification. Primary 55M20, 57Rxx.

(C)2009 American Mathematical Society 
The normal bundle of an immersion $h: Y \rightarrow X$ will be denoted $\nu(h)$. For a smooth manifold $X$, the stable normal bundle will be denoted $\nu(X)$ and the tangent bundle will be denoted $\tau(X)$.

The invariant we construct will be an element of a framed bordism group of the following type.

Definition 3. Given a space $X$ and a bundle $\eta$ over $X$, the $n$-dimensional framed bordism group of $X$ with coefficients in $\eta, \Omega_{n}^{f r}(X ; \eta)$, is the bordism group of $n$ dimensional manifolds mapping to $X$ together with stable bundle isomorphisms of the normal bundle to the pullback of $\eta$. More precisely, elements are represented by triples $\left(Y^{n}, h, \bar{h}\right)$, where $Y$ is a closed $n$-dimensional manifold, $h: Y \rightarrow X$, and $\bar{h}: \nu(Y) \rightarrow h^{*} \eta$ is a stable bundle isomorphism. Two elements $(Y, h, \bar{h})$ and $\left(Y^{\prime}, h^{\prime}, \bar{h}^{\prime}\right)$ are framed bordant if there exists a triple $(W, H, \bar{H})$ such that $W$ is a compact $(n+1)$-dimensional manifold with $\partial W=Y \amalg Y^{\prime}, H: W \rightarrow X$ is a map extending $h$ and $h^{\prime}$, and $\bar{H}: \nu(W) \rightarrow H^{*} \eta$ is a stable bundle isomorphism extending $\bar{h}$ and $\bar{h}^{\prime}$.

Our construction will require the following result of Koźniewski [6. Proposition 6] concerning $B$-manifolds. Given a smooth manifold $B$, a $B$-manifold $X$ is a manifold $X$ together with a locally trivial submersion $p: X \rightarrow B$. A $B$-map is a smooth fiber-preserving map.

Lemma 4. Let $X$ and $Y$ be $B$-manifolds, let $Z$ be a $B$-submanifold of $Y$, and let $g: X \rightarrow Y$ be a B-map. Then there is a B-map $g_{1}: X \rightarrow Y$ such that $g_{1}$ is smoothly $B$-homotopic to $g$ and $g_{1}$ is transverse to $Z$.

Our proof relies heavily on a theorem of Hatcher and Quinn [4, Theorem 4.2]. In the theorem, $E_{M}, E_{Q}$ and $E_{P}$ are smooth fiber bundles over a compact manifold $R$ with fibers $M, Q$ and $P$, respectively. Recall that $E\left(i_{P}, i_{Q}\right)$ is the homotopy pullback.

Theorem 5 (Hatcher and Quinn). Let $P^{p}, Q^{q}$ be closed manifolds, $M^{m}$ a manifold, and $R^{k}$ a compact manifold. Assume that there is a diagram

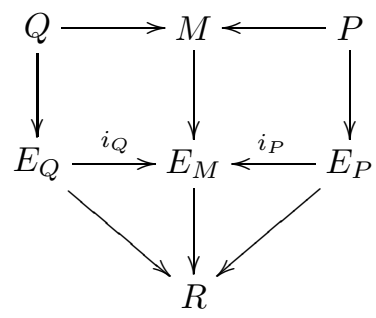

such that $i_{P}$ and $i_{Q}$ are bundle maps which are immersions (embeddings) in each fiber. Suppose $(N, h, \bar{h})$ and $\left(i_{P} \pi i_{Q}, i, \bar{i}\right)$ both represent elements of

$$
\Omega_{p+q+k-m}^{f r}\left(E\left(i_{P}, i_{Q}\right) ; \nu\left(E_{P}, E_{M}\right) \oplus \nu\left(E_{Q}, E_{M}\right) \oplus \nu\left(E_{M}\right)\right) .
$$

Assume further that $m>p+(q+k) / 2+1, m>q+(p+k) / 2+1$ and $p, q>0$. Then $i_{Q}$ is fiber regularly homotopic (isotopic) to a fiber immersion (embedding) $i_{Q}^{\prime}$ with $i_{P} \pi i_{Q}^{\prime}=N$ if and only if $\left[i_{P} \pi i_{Q}\right]=[N]$ is zero in $\Omega_{p+q+k-m}^{f r}\left(E\left(i_{P}, i_{Q}\right) ; \nu\left(E_{P}, E_{M}\right)\right.$ $\left.\oplus \nu\left(E_{Q}, E_{M}\right) \oplus \nu\left(E_{M}\right)\right)$. 


\section{THE INVARIANT}

Let $p: E^{n+k} \rightarrow B^{n}$ be a smooth fiber bundle with compact fibers and $n>2$. Assume that $B$ is a closed manifold. Let $f: E \rightarrow E$ be a map such that $p \circ f=p$ (i.e., a map of fiber bundles over $B$ ).

The fibered product $E \times_{B} E$ is a fiber bundle over $B$ with the fiber over $b \in B$ given by $F_{b} \times F_{b}$, where $F_{b}$ is the fiber of $p$ over $b$. The dimension of $E \times{ }_{B} E$ is $n+2 k$. Let $\Delta: E \rightarrow E \times_{B} E$ be the diagonal map given by $\Delta(x)=(x, x)$; note that $\Delta$ is a map of fiber bundles over $B$. Let $\Delta_{f}: E \rightarrow E \times_{B} E$ be the map of fiber bundles given by $\Delta_{f}(x)=(x, f(x))$.

Take the homotopy pullback

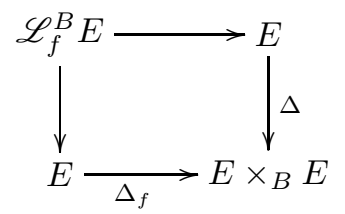

By Lemma 4. $\Delta_{f}$ is fiber homotopic to a map $\Gamma: E \rightarrow E \times_{B} E$ such that $\Gamma$ is transverse to $\Delta$. Take the transverse pullback

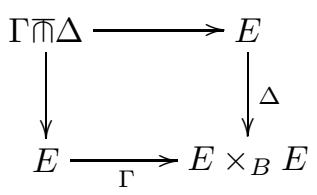

By universality of the homotopy pullback, there is a map $\Gamma \pi \Delta \rightarrow \mathscr{L}_{f}^{B} E$. By transversality, $\nu(\Gamma \pi \Delta)$ is stably isomorphic to $\nu(B)$. Thus, we get that

$$
[\Gamma \pi \Delta] \in \Omega_{n}^{f r}\left(\mathscr{L}_{f}^{B} E ; \nu(B)\right) .
$$

Definition 6. The Lefschetz invariant is

$$
L(f)=[\Gamma \pi \Delta] \in \Omega_{n}^{f r}\left(\mathscr{L}_{f}^{B} E ; \nu(B)\right) .
$$

It is easy to see that this invariant is well-defined and is an extension of the Lefschetz-Nielsen invariant $L(f, *, \tau)$.

\section{The PARAMETRIZED FIXED POINT THEOREM}

Let $f: E \rightarrow E$ be defined as in the last section.

Theorem 7 (Parametrized Fixed Point Theorem). If $f$ is fiber homotopic to a map with no fixed points, then $L(f)=0$. If $k>n+2$ and $L(f)=0$, then $f$ is fiber homotopic to a map with no fixed points.

Proof. Assume $f$ is homotopic to $f^{\prime}$ such that $\operatorname{Fix}\left(f^{\prime}\right)=\emptyset$. Then $\Delta_{f^{\prime}} \cap \Delta$ is zero bordant, and so $\Delta_{f}, \pi \Delta$. By definition, then,

$$
L(f)=[\emptyset]=0 \in \Omega_{n}^{f r}\left(\mathscr{L}_{f}^{B} E, \nu(B)\right) .
$$


For the converse, assume $L(f)=0$. Note that $k>n+2$ gives the dimension requirements, so that we can apply Theorem 5 to the following diagram:

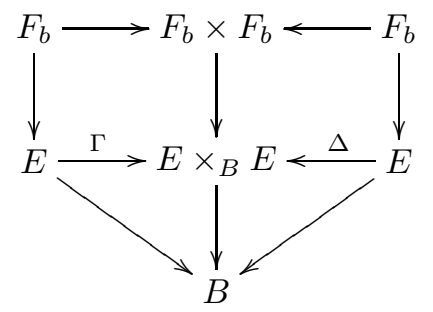

which gives a fiber homotopy (over $B$ ) of $\Gamma$ to $\Gamma^{\prime}: E \rightarrow E \times{ }_{B} E$ such that $\operatorname{im}\left(\Gamma^{\prime}\right) \cap$ $\operatorname{im}(\Delta)=\emptyset$.

It remains to show that $\Gamma^{\prime}$ is fiber homotopic to a map of the form $\Delta_{f^{\prime}}$ such that $\operatorname{im}\left(\Delta_{f^{\prime}}\right) \cap \operatorname{im}(\Delta)=\emptyset$. This is done by considering the following diagram:

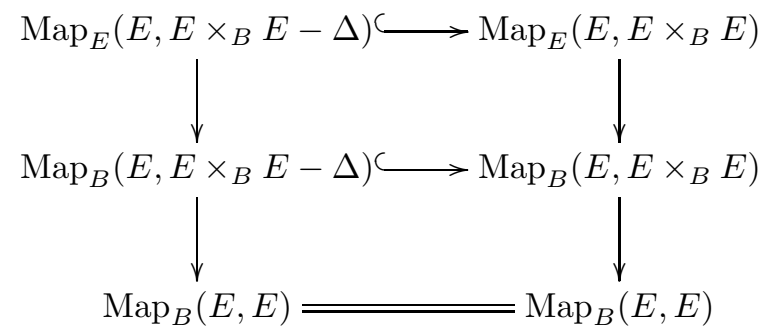

By Fadell [2, the columns are fiber bundle sequences. This implies that the upper square is a homotopy pullback. Thus, since we have $\Delta_{f} \in \operatorname{Map}_{E}\left(E, E \times_{B} E\right)$ and $\Gamma^{\prime} \in \operatorname{Map}_{B}\left(E, E \times_{B} E-\Delta\right)$ such that $\Delta_{f} \simeq \Gamma^{\prime}$ in $\operatorname{Map}_{B}\left(E, E \times_{B} E\right)$, it follows that there is a map

$$
\Gamma^{\prime \prime} \in \operatorname{Map}_{E}\left(E, E \times_{B} E-\Delta\right)
$$

such that $\Gamma^{\prime} \simeq \Gamma^{\prime \prime}$ in $\operatorname{Map}_{B}\left(E, E \times_{B} E-\Delta\right)$. Further,

$$
\Gamma^{\prime \prime} \in \operatorname{Map}_{E}\left(E, E \times_{B} E\right)
$$

implies that $\Gamma^{\prime \prime}$ is of the form $\Delta_{f^{\prime}}$ for some $f^{\prime}: E \rightarrow E$ such that $f$ is fiber homotopic to $f^{\prime}$ and $f^{\prime}$ has no fixed points.

\section{REFERENCES}

1. Vesta Coufal. A Family Version of Lefschetz-Nielsen Fixed Point Theory. Ph.D. thesis, University of Notre Dame, Notre Dame, Indiana, 2004.

2. Edward Fadell. Generalized normal bundles for locally-flat imbeddings. Trans. Amer. Math. Soc., 114:488-513, 1965. MR0179795 (31:4037)

3. Ross Geoghegan. Nielsen fixed point theory. In Handbook of geometric topology, pages 499-521. North-Holland, Amsterdam, 2002. MR1886677 (2003c:55003)

4. Allen Hatcher and Frank Quinn. Bordism invariants of intersections of submanifolds. Trans. Amer. Math. Soc., 200:327-344, 1974. MR0353322 (50:5806)

5. John R. Klein and E. Bruce Williams. Homotopical intersection theory. I. Geom. Topol., 11:939977, 2007. MR2326939 (2008g:55011)

6. Tadeusz Koźniewski. The category of submersions. Bull. Acad. Polon. Sci. Sér. Sci. Math., 27(3-4):321-326, 1979. MR.552057 (81a:57031)

Department of Mathematics, Gonzaga University, Spokane, Washington 99258

E-mail address: coufal@gonzaga.edu 